\title{
Dalteparin in Newborn Thrombosis, Time for a New Starting Dose
}

\author{
Frouwke Steenman $^{a} \quad$ Daniel C. Vijlbrief ${ }^{a} \quad$ Albert Huisman $^{b} \quad$ Marc Bierings $^{c}$ \\ aDepartment of Neonatology, University Medical Center Utrecht/Wilhelmina Children's Hospital, Utrecht University, \\ Utrecht, The Netherlands; ${ }^{\text {b}}$ Department of Clinical Chemistry and Hematology, University Medical Center Utrecht/ \\ Wilhelmina Children's Hospital, Utrecht University, Utrecht, The Netherlands; 'Department Pediatric Hematology \\ and Stem Cell Transplantation, University Medical Center Utrecht/Wilhelmina Children's Hospital, Utrecht \\ University, Utrecht, The Netherlands
}

\author{
Keywords \\ Dalteparin · Low-molecular-weight heparin · Thrombosis . \\ Dosing
}

\begin{abstract}
Background: Neonatal thrombosis is a frequently encountered complication in a neonatal intensive care unit. Dalteparin can be used to treat thrombosis in newborn infants. $\mathbf{O b}$ jectives: In this study, we evaluate the current recommended starting dose of $129 \pm 43 \mathrm{U} / \mathrm{kg} / 24 \mathrm{~h}$, hypothesizing that this dose is too low to reach therapeutic anti-Xa levels. Methods: From 2008 until 2017, all infants treated with dalteparin in the University Medical Centre Utrecht were included in this study. In this retrospective cohort study, the correlation between dose and anti-Xa level was observed. Results: Sixty-six infants were included. The most common thrombus types were catheter-related (29 patients, $44 \%$ ) and venous sinus thrombosis (28 patients, 43\%). The mean dalteparin dose needed for the first adequate anti-Xa level $(0.5-1.0 \mathrm{IU} / \mathrm{mL})$ was $297.6 \mathrm{U} / \mathrm{kg} / 12 \mathrm{~h}$. Two infants developed a first bleeding episode under dalteparin therapy; they both had anti-Xa levels in the therapeutic range. Conclusion: The increase of the starting dose of dalteparin will lead to earlier therapeutic levels of anti-Xa in the studied population and appears to be safe. However, this needs to be evaluated in further study.

(c) 2021 The Author(s)

Published by S. Karger AG, Basel
\end{abstract}

karger@karger.com www.karger.com/neo

Karger"

GOPEN ACCESS
(C) 2021 The Author(s)

Published by S. Karger AG, Basel

This is an Open Access article licensed under the Creative Commons Attribution-NonCommercial-4.0 International License (CC BY-NC) (http://www.karger.com/Services/OpenAccessLicense), applicable to the online version of the article only. Usage and distribution for commercial purposes requires written permission.

\section{Introduction}

Neonatal thrombosis is a frequently encountered complication in a neonatal intensive care unit (NICU) with a reported incidence of 2.4-6.6 per 1,000 NICU admissions [1]. Risk factors for thrombosis in neonates are intravascular catheters, perinatal asphyxia, systemic infections, and pro-thrombotic factors [1-4]. Low-molecular-weight heparin (LMWH) is the therapy of choice. Doses are adjusted using anti-Xa level measurements, aiming at levels between 0.5 and $1.0 \mathrm{U} / \mathrm{mL}$ [5].

To the best of our knowledge, no data were reported on the use of dalteparin in preterm infants. It is well known that the infants' hemostatic system differs from that of adults, maturing until the age of 6 months [6]. The pharmacokinetics and pharmacodynamics of LMWHs are likely to differ from older children and adults, suggesting a higher dose for neonates and younger infants [7, 8]. This difference in coagulation physiology emphasizes the need to evaluate the efficacy of (starting) doses of LMWH in neonates of different gestational ages (GAs).

\section{Materials and Methods}

We report on a retrospective cohort study of all infants admitted to the NICU of the University Medical Center Utrecht between January 2010 and January 2017, who were treated with dalteparin,

Daniel C. Vijlbrief

Department of Neonatology

University Medical Center Utrecht/Wilhelmina Children's Hospital

Lundlaan 6, NL-3584 EA Utrecht (The Netherlands)

D.C.Vijlbrief@umcutrecht.nl 
Table 1. Dose adjustment protocol for dalteparin based on anti-Xa levels (adopted from local protocol)

\begin{tabular}{cll}
\hline & Dose adjustment & Next anti-Xa sample \\
\hline $0.0-0.24, \mathrm{U} / \mathrm{mL}$ & Increase dose by $50 \%$ & After the second new dose \\
$0.25-0.49, \mathrm{U} / \mathrm{mL}$ & Increase dose by $25 \%$ & After the second new dose \\
$0.5-1.20, \mathrm{U} / \mathrm{mL}$ & No adjustment necessary & Weekly in the first month, then monthly \\
$1.21-1.50, \mathrm{U} / \mathrm{mL}$ & Decrease dose by $25 \%$ & After the second new dose \\
$>1.5, \mathrm{U} / \mathrm{mL}$ & Skip a dose then decrease dose by $50 \%$ & After the second new dose \\
\hline
\end{tabular}

Table 2. Dosing and response to treatment

$\begin{array}{lc}\text { Start dose, U/kg } & 185(123-200) \\ \text { Start anti-Xa level, U/mL } & 0.26(0.18) \\ \text { First effective dose, U/kg } & 289(231-362) \\ \text { First effective anti-Xa level, U/mL } & 0.60(0.55-0.75) \\ \text { Days until first effective dose, d } & 3.0(1.0-5.0) \\ \text { Dose at discharge, U/kg } & 315(89) \\ \text { Anti-Xa level at discharge, U/mL } & 0.63(0.26) \\ \text { Dose adjustments, } n & 3.0(1.8) \\ \text { Dalteparin treatment, } n, \mathrm{~d} & 73.0(17.0-90.0)\end{array}$

Data are represented as mean with SD or median with IQR, where appropriate. IQR, interquartile range; SD, standard deviation.

the first-line treatment for thrombosis in our unit. We defined preterm birth as GA between 32 and 37 weeks, very preterm as GA between 28 and 32 weeks, and extreme preterm as GA less than 28 weeks. Subjects were identified by anti-Xa level measurements in the hospital laboratory for clinical chemistry and hematology. Since this was a retrospective study using anonymized patient information, approval by an ethical review board (METC, UMC Utrecht, 18-077C) was waived. We analyzed data for GA, sex, age at diagnosis, use of catheters, presence of infection at diagnosis, follow-up, and complications, mainly bleeding incidents. Anti-Xa levels, concerning the dose of dalteparin, were analyzed until resolution of the thrombus or the end of treatment. Statistical analysis was done using SPSS. Categorical variables were summarized using frequencies and percentages. Continuous variables were summarized using means and standard deviation in case of a normal distribution. Otherwise, medians and interquartile ranges (IQRs) were used. To compare doses between infants, we used the dose divided by the weight. The doses were administered twice daily, following local protocol.

Treatment was started when the thrombosis was symptomatic (persistent thrombocytopenia, signs of occlusion, hypertension), intracardial propagation, or occlusion of a major vessel was found. The local protocol advised a starting dose between 100 and $200 \mathrm{U} /$ $\mathrm{kg} /$ dose twice daily administered subcutaneously (s.c.). This recommendation is based on the 2012 CHEST Guidelines for antithrombotic therapy in neonates [5]. Cranial ultrasound was performed routinely before initiation of treatment and then at least weekly until discharge. For venous sinus thrombosis, an MRI was performed for diagnosis and repeated before cessation of dalteparin therapy after 3 months. Routine blood samples for hemoglobin, thrombo- cytes, and electrolytes were part of routine clinical care during admission to the unit, and after discharge, they were sampled with the anti-Xa levels. If thrombocytopenia occurred, patients received thrombocyte transfusion according to local protocol.

Anti-Xa levels were measured (using the Liquid anti-Xa assay on the STA-R evolution coagulation analyzer [Diagnostica Stago, Asnieres-sur-Seine, France]) $4 \mathrm{~h}$ after administration, doses were adjusted according to the schedule from local protocol in Table 1. After initiation of therapy, the first sample was taken $4 \mathrm{~h}$ after the second dose. After every dose adjustment, a new anti-Xa level was measured after 2 new doses. With stable levels, weekly levels were measured in the first month, after that, monthly levels were taken.

\section{Results}

Sixty-six patients were included. Fifty-one infants were male $(77 \%)$. Thirty-nine infants were born preterm (59\%), of whom 27 (41\%) very preterm, including 17 (26\%) extremely preterm. The median birth weight was 2,231 g (IQR 975-3,238). The most common thrombus types were catheter-related (29 patients, $44 \%$ ) and venous sinus thrombosis (28 patients, $43 \%$ ). Most thrombi were venous of origin (49 patients, $74 \%$ ), but also arterial (16 patients, $24 \%$ ), and combined (1 patient, $2 \%$ ).

In $47(78.3 \%)$ of the cases, the initial response to treatment was shrinking of the thrombus. In 1 (1.7\%) infant, the thrombus grew during treatment with dalteparin. In $12(20.0 \%)$ cases, no direct change in thrombus size was observed.

The median dose at which the patient reached an antiXa level of 0.5 or higher for the first time was $289 \mathrm{U} / \mathrm{kg} / 12$ $\mathrm{h}$ (IQR 231-362). This was reached in a median of 3.0 days (IQR 1-5). The mean dose that led to an anti-Xa level between 0.5 and 1.0 was $327 \mathrm{U} / \mathrm{kg} / 12 \mathrm{~h}$.

The mean dose required to reach the first effective anti-Xa level was $303 \mathrm{U} / \mathrm{kg} / 12 \mathrm{~h}$ (SD: 74) in premature infants as opposed to 296 (SD: 102) in term infants ( $p=$ $0.15)$. The very premature infants required a mean dose of $367 \mathrm{U} / \mathrm{kg} / 12 \mathrm{~h}$ (SD: 100) ( $p=0.71)$, and the extremely premature infants a mean dose of $313 \mathrm{U} / \mathrm{kg} / 12 \mathrm{~h}$ (SD: 101) $(p=0.91)$. 
Table 2 provides more dosing details and results. Twenty-two children had a history of bleeding before the start of LMWH. Of those patients, in 2 patients the bleeding restarted or increased while under treatment (bleeding in a nonrelated hemangioma, propagation of a preexisting intraventricular hemorrhage). The severity of the bleeding, according to the ISTH-criteria, was one instance of major bleeding and one instance of clinically relevant nonmajor bleeding. In one patient, the anti-Xa was 1.16 , potentially explaining the bleeding.

In 2 patients, a first bleeding episode developed under LMWH treatment, again one instance of major bleeding and one instance of clinically relevant nonmajor bleeding (subcapsular hematoma of the kidney following a renal arterial thrombosis, upper leg hematoma). They both had anti-Xa levels in the therapeutic range.

Two patients died while under treatment, primarily due to the consequences of their thrombosis. Sixteen patients $(25 \%)$ had lifelong sequelae of their thrombosis such as epilepsy, cognitive impairment, or chronic renal insufficiency, indicating the seriousness of their thrombotic events. Forty-five children (71\%) had no persisting damage of their thrombosis. No other side effects from the treatment, such as thrombocytopenia, hyperkalemia, or elevated liver enzymes were found.

\section{Discussion/Conclusion}

Our results suggest the need for a higher starting dose of dalteparin in very ill neonates presenting with thrombosis. The mean effective dose (according to anti-Xa levels) in our cohort is $327 \mathrm{U} / \mathrm{kg} / 12 \mathrm{~h}$. We found a high interindividual variation in the first effective dose. The number of bleeding events seen in our study (4 cases of increased or initial bleeding) is similar to that seen in other studies in neonatal antithrombotic therapy [5]. Our cohort was possibly too small to identify differences between subgroups of different GA. According to our results, an initial dalteparin dose of $250-300 \mathrm{U} / \mathrm{kg} / 12 \mathrm{~h}$ is suggested as a starting dose to reach adequate anti-Xa levels in these small children with further tailored treatment based on anti-XA levels. Evaluation of the efficacy (both regarding anti-Xa levels, thrombus clearance, and earlier on target) and safety (mainly bleeding) of our suggested approach in larger prospective cohort studies of (very) preterm infants is warranted.

\section{Statement of Ethics}

Since this was a retrospective study using anonymized patient information, approval by an ethical review board (METC, UMC Utrecht, 18-077C) was waived.

\section{Conflict of Interest Statement}

The authors have no conflicts of interest to declare.

\section{Funding Sources}

No funding was received for this study.

\section{Author Contributions}

F.S., D.V., A.H., and M.B. contributed to the design of the study, interpreted the data, drafted the work, and revised it for contend and made a final approval for submission. F.S. and D.V. were reasonable for the acquisition and analysis of the data.

\section{References}

1 Saxonhouse MA. Thrombosis in the neonatal intensive care unit. Clin Perinatol. 2015 Sept 1;42(3):651-73.

2 Schmidt B, Andrew M. Neonatal thrombosis: report of a prospective Canadian and international registry. Pediatrics. $1995 \mathrm{Nov}$ 1;96(5 Pt 1):939-43.

3 Nowak-Gottl U, von Kries R, Gobel U. Neonatal symptomatic thromboembolism in Germany: two year survey. Arch Dis Child Fetal Neonatal Ed. 1997 May 1;76(3):163.

4 Boo NY, Wong NC, Zulkifli SS, Lye MS. Risk factors associated with umbilical vascular catheter-associated thrombosis in newborn infants. J Paediatr Child Health. 1999 Oct 1;35(5):460-5.
5 Monagle P, Chan AKC, Goldenberg NA, Ichord RN, Journeycake JM, Nowak-Göttl U, et al. Antithrombotic therapy in neonates and children: antithrombotic therapy and prevention of thrombosis, 9th ed: American college of chest physicians evidence-based clinical practice guidelines. Chest. 2012 Feb 1; 141(Suppl 2):e737S-801S.

6 Toulon P. Developmental hemostasis: laboratory and clinical implications. Int J Lab Hematol. 2016 May 01;38(Suppl 1):66-77.
7 Nowak-Göttl U, Bidlingmaier C, Krümpel A Göttl L, Kenet, G. Pharmacokinetics, efficacy, and safety of LMWHs in venous thrombosis and stroke in neonates, infants and children. Br J Pharmacol. 2008 Oct 1;153:1120-27.

8 Cvirn G, Hoerl G, Tafeit E, Heinl N, Wodrig $\mathrm{K}$, Wagner T, et al. Effects of nadroparin, enoxaparin, and unfractionated heparin on endogenous formation of factor $\mathrm{Xa}$ and IIa and on thrombelastometry profiles in cord versus adult blood. Neonatology. 2011; 100(1):23-31. 